\title{
IRREVERSIBILITY IN NON-LINEAR OSCILLATOR SYSTEMS $\uparrow$
}

\author{
JOSEPH FORD \\ School of Physics, Georgia Institute of Technology
}

\begin{abstract}
The statistical behaviour of individual phase-space trajectories for non-linear oscillator systems is demonstrated via computer calculations. These results are interpreted in terms of a mathematical theorem due to A. N. Kolmogorov, V. I. Arnol'd and J. Mojer.
\end{abstract}

\section{INTRODUCTION}

In this paper we investigate the classical motion of oscillator systems governed by the Hamiltonian

$$
H=\sum_{k=1}^{N} \frac{1}{2} \omega_{k}\left(P_{k}^{2}+Q_{k}^{2}\right)+\gamma\left[V_{3}+V_{4}+\ldots\right]
$$

where $N$ is the number of oscillators, $\omega_{k}$ are the positive frequencies of the harmonic approximation, $\gamma$ is the non-linear coupling parameter, and $V_{3}, V_{4}$, etc., are cubic, quartic, etc., polynomials in $Q_{k}$ and $P_{k}$. Our intent is to determine those essential properties of Hamiltonian 1 which are crucial for irreversibility. In particular, we focus our attention on the individual trajectories of Hamiltonian 1 and seek to determine when most of these trajectories exhibit stochastic behaviour, by which we mean that a trajectory moves more or less randomly over a sizeable part, perhaps all, of the energy surface. From the viewpoint of thermodynamics, clearly most $\left(Q_{k}, P_{k}\right)$ sets on a widely stochastic trajectory would correspond to equilibrium. Thus starting the trajectory at a disequilibrium $\left(Q_{k}, P_{k}\right)$ set would inevitably lead to equilibrium giving the appearance of irreversibility. Indeed, computer calculations for simple examples of Hamiltonian 1 show that, under the proper conditions, the approach to equilibrium is rapid and large deviations from equilibrium are rare.

It is perhaps most convenient to discuss the stochastic properties of Hamiltonian 1 in terms of a theorem due to Arnol'd ${ }^{1}$. Arnol'd rigorously proves that most (in the sense of measure theory) trajectories for Hamiltonian 1 lie on smooth, $N$-dimensional, integral surfaces (called tori) embedded in the $2 \mathrm{~N}$-dimensional phase space provided, among other things, that: (1) either $\gamma$ or, equivalently, the total energy is sufficiently small, and

$\dagger$ This work supported in part by the National Science Foundation. 
(2) the harmonic frequencies do not satisfy resonant frequency conditions of the form $\sum_{k=1}^{N} n_{k} \omega_{k}=0$ when the integers $n_{k}$ are such that $\sum_{k=1}^{N}\left|n_{k}\right| \leqslant 4$.

Clearly when Conditions (1) and (2) are satisfied most trajectories are not widely stochastic. The virtue of the Arnol'd theorem for our purposes is that, to a large extent, it actually delimits the conditions for stochastic behaviour. Indeed violation of Condition (1) even for $N=2$ in general leads to a rather sudden onset of widespread stochasticity ${ }^{2-4}$ as the non-linearity becomes strong. Violation of Condition (2) for $N \geqslant 3$ leads to widespread stochasticity even in the limit as the non-linearity tends to zero ${ }^{5}$.

Conditions (1) and (2), when satisfied, yield non-stochastic motion because they minimize the effects of resonant interactions. In the langage of quantum mechanics, Condition (2), for $N \geqslant 3$, disallows those resonant, three and four phonon processes so widely invoked in solid state physics ${ }^{6}$. Condition (1) does not allow, the higher order phonon processes to affect more than a minority (in the sense of measure theory) of states. On the other hand, violation of Condition (1) and/or Condition (2) gives the non-linear resonances free rein to affect the motion. In order to illustrate these effects, in Section II we demonstrate by example for $N=2$ that each isolated resonant interaction serves to introduce new stable and unstable periodic orbits into the unperturbed classical motion. When two or more resonances overlap and influence the same trajectory, the system phase space trajectory wanders over the energy surface being scattered, in a sense, by the randomly positioned stable and unstable periodic orbits. In Section III, we demonstrate, again using a simple example, that overlapping, cubic, resonant 'three phonon' interactions can yield stochastic behaviour even in the limit as the nonlinearity tends to zero. Section IV then presents our conclusions.

In this paper we actually demonstrate stochasticity only for simple examples and for small $N$; moreover, we rely heavily on computer calculations. We do this without apology. Indeed we wish to emphasize that irreversibility is not a property requiring infinite $N$, that irreversibility can be illustrated using simple examples, and that the computer can perform highly informative 'experiments'. Indeed it is the author's belief that the computer, guided by analytical considerations, can contribute heavily to our understanding of statistical mechanics through the detailed study of small systems.

\section{STOCHASTICITY FOR $N=\mathbf{2}$}

The stochasticity of Hamiltonian 1 for relatively strong non-linearity can be illustrated by studying some simple examples for the case $N=2$. Let us begin by considering the isolated resonancet described by

$$
H=J_{1}+J_{2}-J_{1}^{2}-3 J_{1} J_{2}+J_{2}^{2}+\beta J_{1} J_{2}^{\frac{3}{2}} \cos \left(2 \varphi_{1}-3 \varphi_{2}\right)
$$

where $\beta$ is chosen such that the resonant, angle term is small relative to the

$\dagger$ Hamiltonians 2 and 3 actually violate Condition (2) of Arnol'd's theorem without yielding stochastic behaviour. When Condition (2) is violated using only a single or isolated resonance interaction the system motion is non-stochastic because an isolated resonance always yields motion on smooth, integral surfaces. 
pure- $J$ terms. Hamiltonian 2 has for convenience been expressed in actionangle variables and the cosine term represents the resonant 'five phonon' interaction $2 \Omega_{1} \rightleftarrows 3 \Omega_{2}$. Here $\Omega_{1}$ and $\Omega_{2}$ are the initial condition dependent, non-linear frequencies of the motion for $\beta=0$. When $N=2$, it is possible to completely survey the motion generated by the Hamiltonian using graphical methods, and we now describe these methods and present results.

Since the Hamiltonian $H$ is a constant of the motion, each system trajectory in the four-dimensional phase space is confined to a three-dimensional subspace. Let us now imagine that this three-dimensional subspace is intersected by a two-dimensional plane, called a level curve plane $e^{2.4}$. If a system trajectory is stochastic then its intersections with this plane will consist of a set of randomly scattered points. If the system trajectory is nonstochastic, i.e. the system trajectory lies on a two-dimensional integral surface, then the intersection points with the plane will form a curve, called a level curve. Plotting the level curve plane intersections for a representative sampling of trajectories will thus reveal the general character of the motion. In Figure 1, we plot a typical level curve diagram for Hamiltonian 2. Were

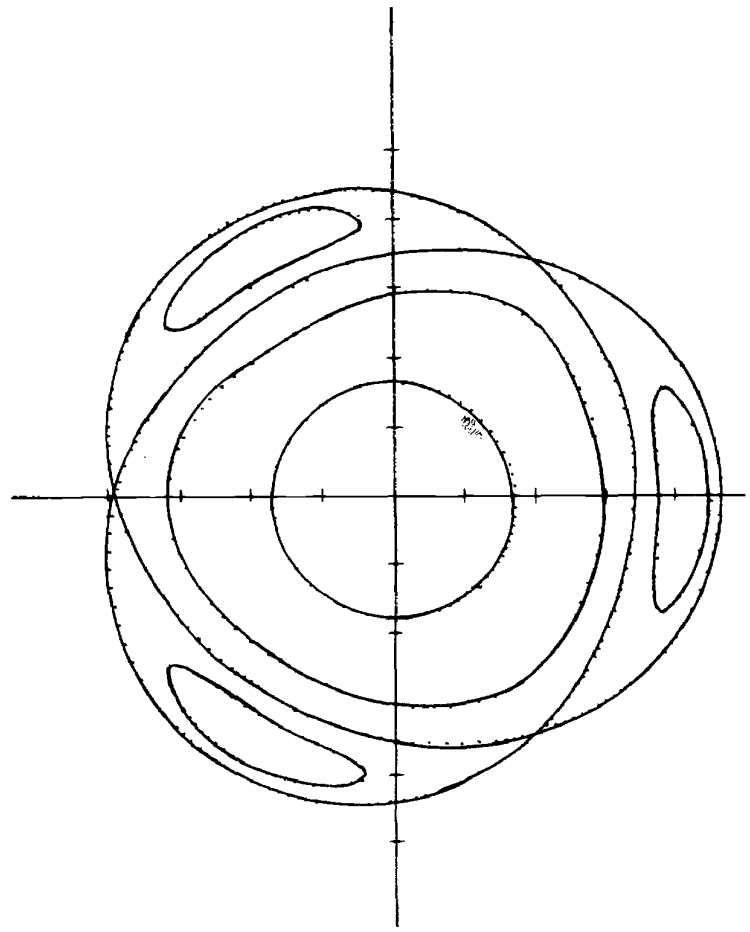

Figure 1. Typical level curves for Hamiltonian 2 showing the distortion in the $\beta=0$ level curves due to an isolated resonance.

$\beta=0$, the level curves would be circles centred on the origin; the $2 \Omega_{1} \rightleftarrows 3 \Omega_{2}$ resonant interaction distorts the $\beta=0$ level curves in a relatively narrow region of the plane by introducing the new stable (three central invariant points of the crescent regions) and unstable (three self-intersection points) 
periodic orbits shown. The level curve plane for Hamiltonian 2 exhibits only non-stochastic trajectories because the additional integral $I=3 J_{1}+2 J_{2}$ confines all system trajectories to lie on smooth, two-dimensional integral surfaces.

Next we consider the isolated resonance described by

$$
H=J_{1}+J_{2}-J_{1}^{2}-3 J_{1} J_{2}+J_{2}^{2}+\alpha J_{1} J_{2} \cos \left(2 \varphi_{1}-2 \varphi_{2}\right)
$$

where the pure- $J$ terms are the same as in Hamiltonian 2 and where $\alpha$ is chosen such that the resonant angle dependent term is small relative to the pure- $J$ terms. This angle dependent term represents the resonant 'four phonon' interaction $2 \Omega_{1} \rightleftarrows 2 \Omega_{2}$. A typical level curve plane for Hamiltonian 3 appears in Figure 2. Here again the resonant interaction has distorted the

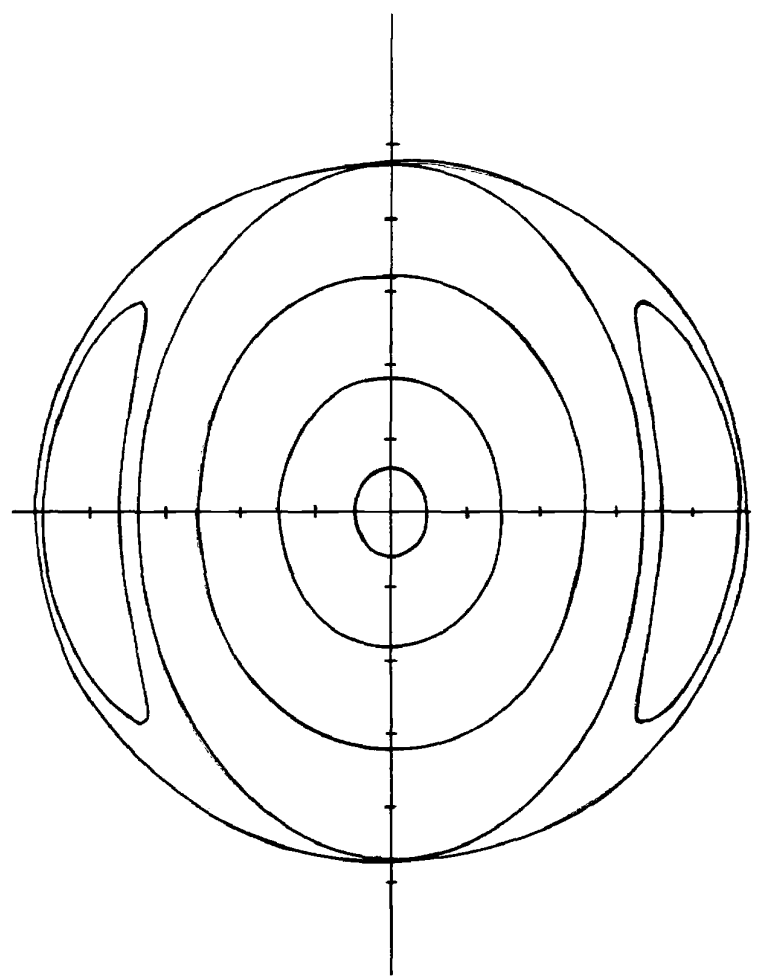

Figure 2. Typical level curves for Hamiltonian 3 showing the distortion of the $\alpha=0$ level curves for an isolated resonance.

$\alpha=0$ motion by introducing stable and unstable periodic orbits, but located in a different part of the plane. Also here again the additional integral $I=J_{1}+J_{2}$ ensures smooth level curves everywhere. In Hamiltonians 2 and 3 as $\alpha$ and $\beta$ increase from zero, the widths of the resonant, crescent regions also increase from zero. Consequently, one anticipates that if both 
interactions acted simultaneously they might overlap for $\alpha$ and $\beta$ sufficiently large. We thus now consider the doubly-resonant Hamiltonian

$$
\begin{aligned}
H=J_{1}+J_{2}-J_{1}^{2}-3 J_{1} J_{2}+J_{2}^{2}+\alpha J_{1} J_{2} & \cos \left(2 \varphi_{1}-2 \varphi_{2}\right) \\
& +\beta J_{1} J^{\frac{3}{2}} \cos \left(2 \varphi_{1}-3 \varphi_{2}\right)
\end{aligned}
$$

In Figure 3 we show the computer obtained level curve diagram typical of Hamiltonian 4 when $\alpha$ and $\beta$ are small. Figure 4 shows the computer calcu-

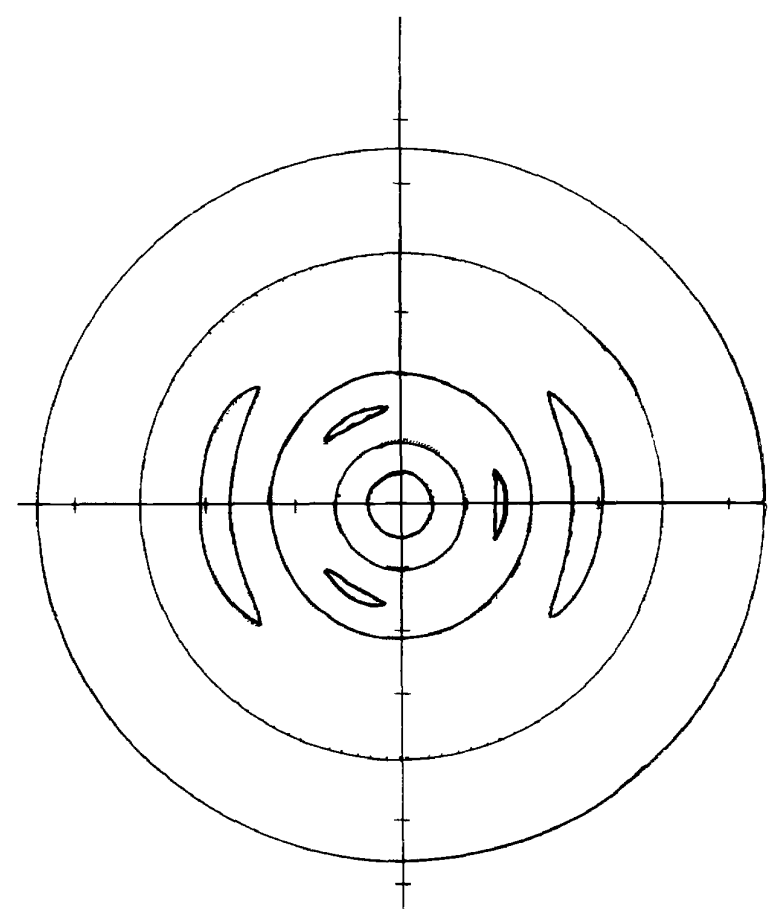

Figure 3. Typical level curves for Hamiltonian 4 for relatively small $\alpha$ and $\beta$. Here the two resonance regions do not overlap.

lated level curve diagram for $\alpha$ and $\beta$ sufficiently large that resonance overlap occurs. The isolated dots represent intersection points for a single, stochastic trajectory. As $\alpha$ and $\beta$ are increased further or as the energy is increased, the stochastic zone increases in size until it almost completely covers the allowed regions of the plane. This stochasticity, due to resonance overlap, here illustrated for the simple Hamiltonian 4 is in general characteristic of Hamiltonian 1 when the non-linearity is large. 


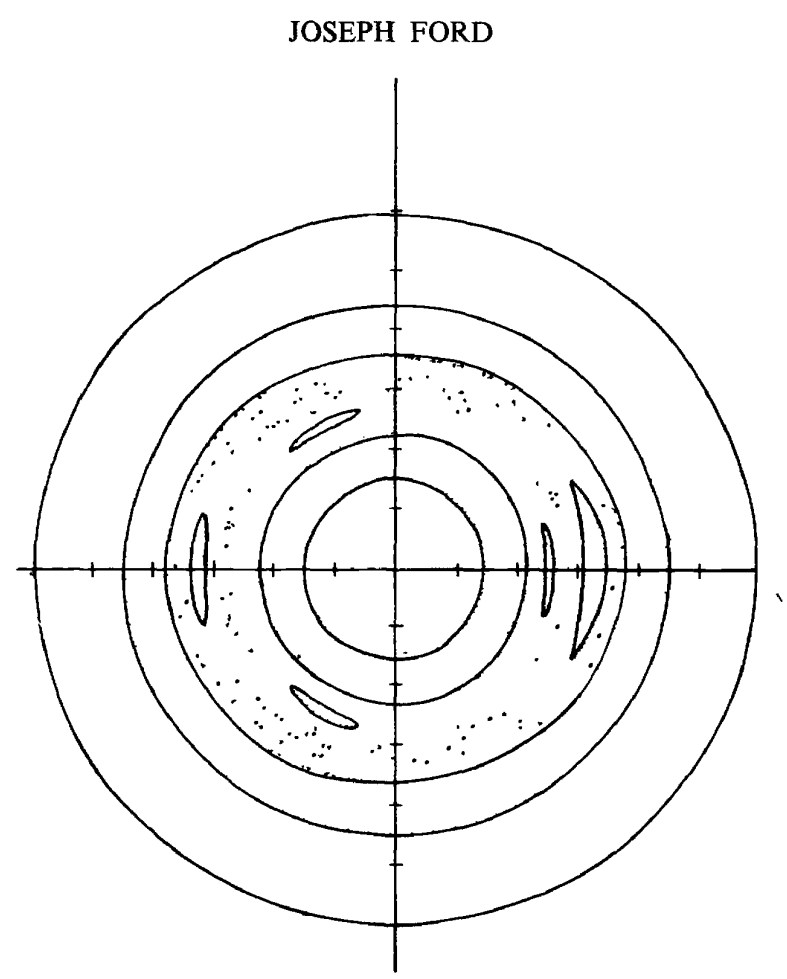

Figure 4. Level curves for Hamiltonian 4 for values of $\alpha$ and $\beta$ such that overlap occurs. The isolated dots are the intersection points for a single trajectory.

\section{STOCHASTICITY FOR $N=\mathbf{3}$}

In order to illustrate that stochasticity can occur for Hamiltonian 1 even in the limit as the nonlinearity goes to zero provided $N \geqslant 3$ and Condition (2) is violated, we choose to consider the doubly-resonant, three particle Hamiltonian

$$
\begin{aligned}
H=J_{1}+2 J_{2}+3 J_{3}+\gamma\left[\alpha J_{1} J_{2}^{\frac{1}{2}}\right. & \cos \left(2 \varphi_{1}-\varphi_{2}\right) \\
& +\beta\left(J_{1} J_{2} J_{3}\right)^{\frac{1}{2}} \cos \left(\varphi_{1}+\varphi_{2}-\varphi_{3}\right]
\end{aligned}
$$

where the harmonic frequencies $\omega_{1}=\frac{1}{2} \omega_{2}=\frac{1}{3} \omega_{3}=1$ and these non-linear terms were chosen in order to illustrate the effect of resonant 'three phonon' interactions. In particular the $\alpha$-term represents the $2 \omega_{1} \rightleftharpoons \omega_{2}$ process while the $\beta$-term represents the $\left(\omega_{1}+\omega_{2}\right) \rightleftarrows \omega_{3}$ process. In order to show that stochasticity can persist even as the non-linearity goes to zero, i.e. as $\gamma$ goes to zero, we introduce the time dependent canonical transformation

$$
\begin{gathered}
J_{1}=\bar{J}_{1}, J_{2}=\bar{J}_{2}, J_{3}=\bar{J}_{3} \\
\varphi_{1}=\bar{\varphi}_{1}+t, \varphi_{2}=\bar{\varphi}_{2}+2 t, \varphi_{3}=\bar{\varphi}_{3}+3 t
\end{gathered}
$$


Hamiltonian 5 then becomes

$$
\mathscr{H}=\gamma\left[\alpha \bar{J}_{1} \bar{J}_{2}^{\frac{1}{2}} \cos \left(2 \bar{\varphi}_{1}-\bar{\varphi}_{2}\right)+\beta\left(\bar{J}_{1} \bar{J}_{2} \bar{J}_{3}\right)^{\frac{1}{2}} \cos \left(\bar{\varphi}_{1}+\bar{\varphi}_{2}-\bar{\varphi}_{3}\right)\right.
$$

Since $\gamma$ is merely a multiplicative factor in Hamiltonian 7, we see that $\gamma$ affects only the time scale of the motion and not its stochasticity. Moreover, since $I=\bar{J}_{1}+2 \bar{J}_{2}+3 \bar{J}_{3}$ is a constant of the motion for this simple Hamiltonian, we may reduce the problem to one having two degrees of freedom and thence determine stochasticity using level curve diagrams. In Figure 5 we plot typical computer obtained level curves for two different initial

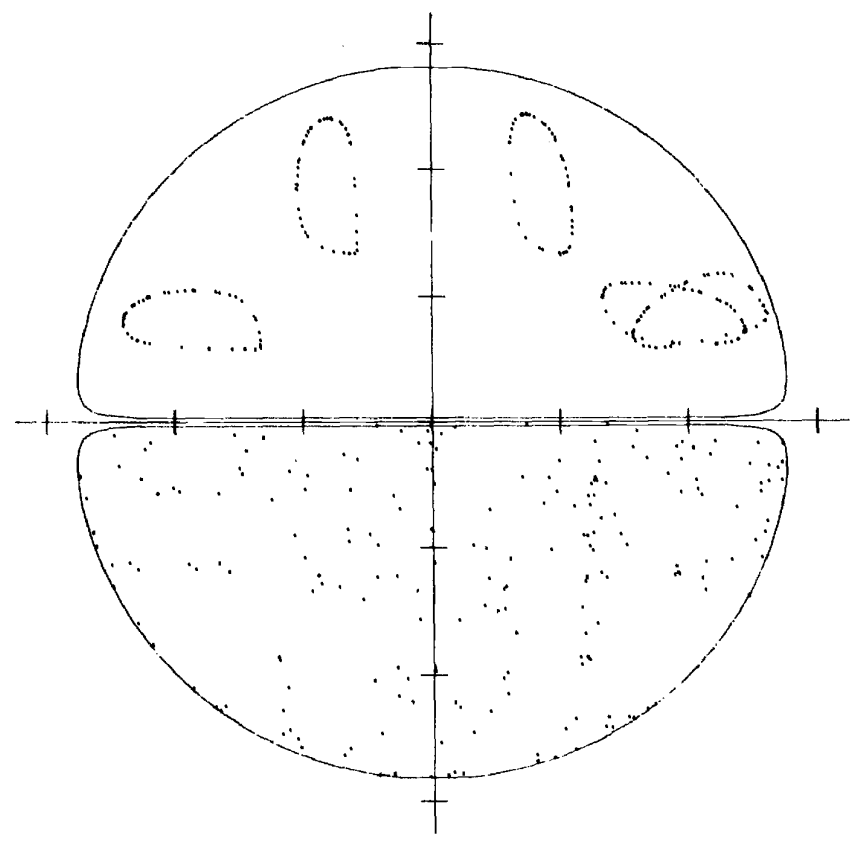

Figure 5. Typical level curves for the reduced Hamiltonian equivalent to Hamiltonian 5. The upper half-plane shows the level curve for one trajectory, and the lower half-plane shows the dots of intersection belonging to another single trajectory. This plane is invariant as $\gamma$ tends to zero.

conditions using the reduced Hamiltonian equivalent to Hamiltonian 5. Since here the level curves only fill a semicircle, we plot the level curve for one trajectory in the upper half-plane and the level curve for another trajectory in the lower half-plane. Since $\gamma$ only determines the time scale for these level curves, Figure 5 is invariant as $\gamma$ tends to zero, excluding $\gamma=0$ of course. The bottom half of Figure 5 exhibits the highly stochastic orbits which can occur. Detailed calculations ${ }^{5}$ reveal that, depending on the ratio $(\alpha / \beta)$, as much as 70 per cent of phase space for Hamiltonian 5 contains stochastic trajectories. Moreover, since the number of overlapping, resonant, cubic interactions increases very rapidly with increasing $\mathrm{N}$, one anticipates that the case $N=3$ yields the minimum stochasticity. 


\section{CONCLUSIONS}

In this brief paper, we have attempted to illustrate, using simple examples, that stochastic behaviour characteristic of all non-linear oscillator systems obeying Hamiltonian 1. Stochasticity arises from resonance overlap which can occur for $N \geqslant 2$. Stochasticity occurs for relatively large non-linearity, almost regardless of the values of the frequencies in the harmonic approximation, due to the overlap of individual, non-linear resonances of various order whose widths are large because the non-linearity is large. For extremely small non-linearity, only the cubic 'three phonon' or possibly the quartic four phonon' resonances can overlap provided $N \geqslant 3$ because the width of these resonances, which depends primarily on the suitably commensurate harmonic frequencies, can remain large even when the non-linearity is small. Said another way, if we specify the system 'state' for these non-linear systems by giving the 'energy' of each oscillator, we observe that an initial individual state is resonantly coupled to that density of final states envisioned by the quantum mechanical Golden Rule ${ }^{7}$ as leading to irreversible behaviour. Moreover, the stochastic irreversibility discussed here is an inherent property of the mechanical equations of motion. This irreversibility occurs provided that there is widespread resonance overlap, and no additional assumptions of a non-mechanical nature are needed. Thus one has here that beginning understanding of the ultimate source of irreversibility which can contribute significantly to statistical mechanics.

\section{REFERENCES}

1 V. I. Arnol'd, Russian Math. Surveys, 18 (No. 6), 85 (1963), Ch. 1, Sec. 9 and 10. Also see V. I. Arnol'd and A. Avez, Ergodic Problems of Classical Mechanics, Ch. 4. W. A. Benjamin: New York (1968).

2 M. Henon and C. Heiles, Astron. J. 69, 73 (1964).

3 F. M. Izrailev and B. V. Chirikov, Dokl. Akad. Nauk S.S.S.R. 166, 57 (1966) [English transl.: Soviet Phys.-Doklady 11, 30 (1966)]. Also see G. M. Zaslavskii. Statistical irreversibility in non-linear systems', Preprint 254, Institute of Nuclear Physics, Novosibirsk, U.S.S.R. (1968); or see N. J. Zabusky and G. S. Deem, J. Computational Phys. 2, 126 (1967).

${ }^{4}$ G. H. Walker and J. Ford, Phys. Rev. 188, 416 (1969).

5 J. Ford and G. H. Lunsford, Phys. Rev. A1, 59 (1970).

6 J. M. Ziman, Electrons and Phonons. Oxford University Press: London (1960).

7 E. Merzbacher, Quantum Mechanics. Wiley: New York (1964). 